\title{
ESTRESSE PERCEBIDO E O USO DE ÁLCOOL, TABACO E OUTRAS DROGAS ENTRE UNIVERSITÁRIOS
}

\section{Luiz Roberto Marquezi Ferro}

Doutorando no Programa de Pós Graduação em Psicologia da Saúde da Universidade Metodista de São Paulo (bolsista CAPES), São Bernardo do Campo (SP). Docente no Centro de Estudo da Arquidiocese de Ribeirão Preto, Brodowski (SP), Brasil.

\section{Álvaro Augusto Trigo}

Mestre em Promoção de Saúde pela Universidade de Franca (Unifran), Franca (SP). Docente no Programa de Ciências da Saúde da Faculdade de Medicina na Universidade de Franca (Unifran), Brasil.

\section{Aislan José Oliveira}

Doutorando no Programa de Pós Graduação em Psicologia da Saúde da Universidade Metodista de São Paulo (bolsista CAPES), São Bernardo do Campo (SP). Docente no Centro Universitário Campos de Andrade (Uniandrade), Curitiba (PR), Brasil.

\section{Marco Aurelio Ramos de Almeida}

Doutorando no Programa de Pós Graduação em Psicologia da Saúde da Universidade Metodista de São Paulo (bolsista CAPES), São Bernardo do Campo (SP). Docente na Universidade Cruzeiro do Sul, São Paulo(SP), Brasil.

\section{Regina Fujiko Tagava}

Doutorando no Programa de Pós Graduação em Psicologia da Saúde da Universidade Metodista de São Paulo, São Bernardo do Campo (SP), Brasil.

\section{Carolina Meneses-Gaya}

Doutora em Saúde Mental pela Faculdade de Medicina da Universidade de São Paulo (USP), Ribeirão Preto(SP) e Pós Doutora pelo Departamento de Psiquiatria e Psicologia Médica pela Universidade Federal de São Paulo, São Paulo(SP), Brasil (UNIFESP), Brasil.

\section{Manuel Morgado Rezende}

Doutor em Saúde Mental pela Universidade Estadual de Campinas(SP) e Pós Doutor em Psicologia da Saúde pela Universidade deAlgarve (Portugal). Docente Titular no Programa de Pós Graduação em Psicologia da Saúde na Universidade Metodista de São Paulo, São Bernardo do Campo (SP), Brasil.
RESUMO: Avaliar a consumo de drogas em uma população de universitários e verificar as associações com estresse percebido foi o objetivo desta pesquisa. A metodologia utilizada foi de pesquisa descritiva com uma população de 373 universitários. A coleta dos dados se deu de forma online; os participantes responderam ao TCLE, o questionário sociodemográfico, o instrumento para mensurar estresse (escala de Estresse Percebido - EP-10) e consumo abusivo de droga (ASSIST). Para verificar a associação entre o uso abusivo de drogas e a variável estudada foi utilizado o modelo de regressão logística com a significância de $\mathrm{p}<0,05$. A regressão logística revelou associação significativa entre uso de tabaco, ter religião e morar com os pais. Para o consumo de álcool e outras drogas a regressão logística apresentou associação com alto estresse. Concluímos que quanto mais os universitários são expostos a fatores de risco, como estresse, maiores as probabilidades para o consumo de drogas.

PALAVRAS-CHAVE: Saúde do Estudante; Transtornos Relacionados ao Uso de Substâncias; Fator de risco; Estresse.

\section{STRESS AND THE USE OF ALCOHOL, TOBACCO AND OTHER DRUGS IN UNDERGRADUATES}

\begin{abstract}
Current study analyzes drug consumption in undergraduates and verifies the association with stress. Methodology involves descriptive research in 373 undergraduates. Data were collected online and participants responded to TCLE, sociodemographic questionnaire, stress measuring tool (Perceived Stress Scale - EP-10) and illicit drug consumption. Logistic regression at $0.05 \%$ significance was employed to assess the association between abuse drugs and the variable under analysis. Logistic regression revealed significant association between tobacco usage, religion and living with parents. In the case of alcohol consumption and other drugs, logistic regression provided association with high stress. The more undergraduates are exposed to risk factors, such as stress, the more their possibilities in drug consumption.
\end{abstract}

PALAVRAS-CHAVE: Student Health; Substance-Related Disorders; Risk Factors; Stress. 


\section{INTRODUÇÃO}

\section{VIDA UNIVERSITÁRIA, DROGAS LÍCITAS E ILÍCITAS}

Na América Latina, jovens entre 18 e 24 anos, especialmente estudantes universitários, apresentam maiores índices de uso de drogas legais e ilegais do que a população geral ${ }^{1}$.

$\mathrm{O}$ ingresso na vida universitária é motivo de satisfação, de inúmeras perspectivas, uma vez que se inicia um mundo desconhecido ${ }^{2}$. Paralelamente, trata-se de um período crítico de maior vulnerabilidade para o início e manutenção do uso de álcool e de outras drogas³. Os novos amigos, a necessidade de autoafirmação, a solidão e o distanciamento dos familiares podem ser fatores conjugados ao abuso de drogas nesse período. O consumo de drogas é comum e frequente entre universitários e esse fenômeno acontece no mundo inteiro, sem distinção entre cursos ${ }^{4}$.

Estudos evidenciam que o álcool é a principal substância de consumo entre os universitários, e isso pode ocorrer principalmente pela maior disponibilidade de acesso, além da bebida alcoólica ser usada como fator de facilitação para ser aceito no grupo ${ }^{2-4}$. Infelizmente cabe ressaltar que essa primazia da prevalência do álcool já se faz notar desde a adolescência, mesmo antes do ingresso na universidade ${ }^{4}$.

Pesquisas demonstram que o ingresso na vida universitária, além de um importante estressor, podem ter considerável significado na iniciação e manutenção do uso de álcool, tabaco e outras substâncias ${ }^{5,6}$.

Particularmente, o aumento do consumo de álcool entre os universitários está associado à diminuição da expectativa de vida dessa população, em decorrência de comportamentos de risco que podem afetar o seu bem-estar, fato que também ocorre com o uso de outras drogas. Cabe registrar que as internações consequentes à dependência de álcool são muito mais numerosas do que aquelas decorrentes da cocaína, por exemplo, apesar das enfáticas manifestações da mídia ${ }^{3}$.

Há um aspecto particular no consumo de álcool que, mesmo de modo isolado, representa um indicador de consumo perigoso, é o padrão de consumo binge ou beber pesado episódico, definido quando o indivíduo ingere grande quantidade de álcool em um período curto de tempo (02 horas). Essa dose é de quatro unidades de álcool para mulheres e cinco unidades para homens, e não é rara sua ocorrência entre os universitários, sobretudo pela participação nas festas denominadas de "open", ${ }^{\text {. }}$.

Sobre as demais substâncias psicoativas, que podem ser alvo de abusos e dependência, mencionamos aquelas com maiores incidências de uso no meio universitário que são a maconha, cocaína (e derivados como crack ) e o outro grupo formado por substâncias sintetizadas em laboratório, como as anfetaminas (e seus numerosos derivados, por exemplo, ecstasy, MDMA ou 3,4-metilenodioxi-metanfetamina), solventes (inalantes), sedativos (barbitúricos, diazepínicos e outros tranquilizantes), alucinógenos, como o LSD (dietilamina do ácido lisérgico), além dos esteroides anabolizantes ${ }^{8}$.

Dentre todas as outras substâncias psicoativas, ilícitas, consideradas como drogas de abuso, e de consumo entre universitários, a maconha é a que apresenta maior frequência de consumo. Segundo os dados da ONU, no seu relatório mundial sobre o Controle de Drogas e Crime, aproximadamente, das duas centenas de milhões de usuários dessas outras substâncias de abuso, 160 milhões consomem maconha, seguidos de 34 milhões que consomem anfetaminas, 16 milhões para opioides e 14 milhões a cocaína, o que torna esses consumos um problema de saúde pública e particularmente de grande relevância para as universidades?

Outro grupo de substâncias que inspira grande preocupação é o das anfetaminas, que são drogas sintéticas. São consumidas tanto na forma de ingestão, como o ecstasy, que é a metilenodioximetanfetamina (MDMA), quando fumadas, como a metanfetamina, que recebe o nome de "ice" e é muito usada na América do Norte. O consumo dessas drogas no Brasil é elevado, não só entre os jovens, estudantes, universitários, mas também por motoristas (conhecida como "rebite"). Entre os estudantes brasileiros do primeiro e segundo graus das dez maiores capitais do país, 4,4\% revelaram já ter experimentado pelo menos uma vez na vida uma droga tipo anfetamina. $\mathrm{O}$ uso frequente (06 ou mais vezes no mês) foi relatado por $0,7 \%$ dos estudantes, com a agravante de que este uso foi mais comum entre as meninas ${ }^{1}$. 


\section{ESTRESSE PERCEBIDO}

A definição de estresse perpassa uma interação entre elementos físicos e psíquicos. O primeiro conceito biológico criado para estresse (bem como o conceito de estressor) foi na década dos anos 50, e define-o como resultado inespecífico de qualquer demanda sobre o corpo, seja de efeito mental ou somático, sendo composto por três fases: alerta, resistência e exaustão ${ }^{10}$. $\mathrm{Na}$ atualidade, Lipp identifica uma fase que ela denomina de "quase-exaustão", de períodos intercalados de bemestar em que o indivíduo resiste, com outros em que surgem doenças pela exaustão do sujeito; ${ }^{(11)}$ na proposta primeira, esse quadro está contido na fase do processo por ele denominada de exaustão, mesmo com a presença de períodos de bem-estar ${ }^{10}$.

Posteriormente, o estresse foi definido como uma reação do organismo, com componentes físicos e/ou psicológicos, causada pelas alterações psicofisiológicas que ocorrem quando o indivíduo se confronta com uma situação que, de um modo ou de outro, o irrite, amedronte, excite ou confunda, ou mesmo que o faça imensamente feliz ${ }^{12}$.

O estresse percebido representa um bom preditor de sintomas físicos e sinaliza maior percepção do indivíduo em termos de sua participação na determinação do seu estado de saúde ${ }^{13}$. É importante notar que a simples presença de fatores estressores, por si só não é suficiente para provocar doença, mas a interação entre eles (estressores), o indivíduo (considerando-se aqui a genética e a capacidade adquirida de gerenciar o estresse) e o ambiente, é que condiciona o resultado final ${ }^{13}$.

Além da multiplicidade de causas concorrentes, considera-se ainda uma relação temporal entre os eventos estressores e o surgimento de sintomas e de transtornos mentais (quanto mais duradouro o estímulo, maiores os riscos). Devemos considerar que na vida universitária (que em geral coincide com a transição da adolescência para a idade adulta), o estudante enfrenta diversos estressores diretamente ligados à nova forma de estudar e aprender, com exigências crescentes inclusive em termos de desempenho, competitividade, concentração de esforços permanente e progressiva, além de outros fatores de estresse decorrentes de responsabilidades tanto acadêmicas quanto pessoais, na criação de relacionamentos mais maduros e estáveis para o seu futuro $^{14}$. Outros aspectos igualmente importantes em termos de estressores, nesse período de universidade, são aqueles decorrentes da mudança de hábitos e estilos de vida, frequentemente diferentes daqueles que o indivíduo trazia de seu ambiente social e familiar, com exigências que podem requerer intenso esforço adaptativo ${ }^{14}$.

Convém mencionar que o uso de álcool, tabaco e substâncias psicoativas pode ser considerado uma das formas de enfrentamento do estresse, comparando-se nessa situação a uma estratégia de coping entendida no seu sentido amplo ${ }^{15}$.

O objetivo dessa pesquisa foi avaliar a consumo de drogas em uma população de universitários e verificar as associações com estresse percebido.

\section{METODOLOGIA}

Tratou-se de um estudo descritivo, com abordagem quantitativa, o que se permitiu testar as hipóteses, obtendo resultados mais concretos e com menos possibilidade de erros interpretativos.

\section{PARTICIPANTES}

Participaram desta pesquisa 373 estudantes de diversos cursos de graduação de uma instituição de ensino superior de uma universidade particular do interior do Estado de São Paulo. Este número de sujeitos é justificado pelo cálculo amostral, de uma população de 12.000 estudantes (dados informados pela secretaria da universidade), com $50 \%$ de heterogeneidade, $5 \%$ de erro amostral e nível de confiança de $95 \%$. Este número de sujeitos nos revela uma amostra de significância. Destes 65,13\% eram mulheres e 34,87\% eram homens, com média de idade de 21 anos (Desvio padrão 4,26).

\section{INSTRUMENTOS}

Utilizaram-se três questionários para a realização desta pesquisa, um que mensurou o consumo de álcool, tabaco e outras drogas, denominado de ASSIST; a Escala de Estresse Percebido (EPS-10) e um questionário sobre 
os dados demográficos, como sexo, idade, curso a que pertencia, estado civil etc.

O ASSIST foi desenvolvido por pesquisadores de vários países sob a coordenação da Organização Mundial de Saúde (OMS), consequentemente, foi traduzido para várias línguas, inclusive para o português do Brasil, já tendo sido testado quanto à sua validade e confiabilidade. É um questionário estruturado contendo oito questões que avaliam o consumo de nove classes de substâncias psicoativas (tabaco, álcool, maconha, cocaína, estimulantes, sedativos, inalantes, alucinógenos, e opiláceos). As questões abordam a frequência de uso na vida e nos últimos três meses, problemas relacionados ao uso, à preocupação a respeito do uso por parte de pessoas próximas ao usuário, ao prejuízo na execução de tarefas esperadas, às tentativas mal sucedidas de cessar ou reduzir o consumo, ao sentimento de compulsão e ao uso por via injetável. Cada resposta corresponde a um escore que varia de 0 a 4 , sendo que a soma total de 0 a 20. Considera-se a faixa de escore de 0 a 3 como indicativa de uso ocasional, de 4 a 15 como indicativa de abuso e acima de 16 como sugestiva de dependência ${ }^{16}$.

A escala de Estresse Percebido (EPS-10) foi traduzida e validada para aplicação no Brasil. As dez perguntas contidas nesta escala indagam a respeito de sentimentos e pensamentos experimentados durante os últimos 30 dias, para assim classificar o nível de estresse percebido pelo respondente ${ }^{17}$. O escore é obtido somando-se os pontos de todas as respostas, após a reversão da pontuação das questões $4,5,7$ e 8 (transformando os valores de 0 para 4; 1 para $3 ; 3$ para 1 ; 4 para 0 e mantendo $2=2$ ). Esse escore total é utilizado como a medida de estresse. É importante ressaltar que o resultado final não é uma medida de critério concorrente, podendo, entretanto, ser comparada a algumas tabelas disponíveis como as da população americana, ou portuguesa $^{(18)}$, ou ainda da validação brasileira com população de professores do sul do Brasil ${ }^{19}$, para a obtenção de um parâmetro relativo aos níveis de estresse no grupo analisado. Assim sendo, estabelecemos com base na pontuação obtida três faixas de estresse: a primeira faixa até 16 pontos que consideramos como de baixo estresse, a segunda faixa de 17 até 21 pontos de estresse médio e a terceira faixa para 22 ou mais, avaliada como de alto estresse ${ }^{12,20 .}$

\section{PROCEDIMENTOS}

O trabalho foi realizado na modalidade de pesquisa online, por meio de uma plataforma no servidor Surveymonkey (http://surveymonkey.com).

A coleta de dados foi realizada entre outubro e novembro de 2013. Os alunos dos cursos de graduação foram informados e convidados a participar da pesquisa em suas salas de aula e pela página da rede social da universidade.

Ao acessarem a pesquisa na plataforma o aluno obtinha informações sobre o estudo de maneira que pudesse decidir ou não participar. Após o aceite, o aluno teve acesso ao Termo de Consentimento Livre e Esclarecido e, em seguida, aos questionários.

Os dados sociodemográficos foram tratados com estatística descritiva. Foi utilizado o modelo de regressão logística para verificar a associação entre o uso abusivo de drogas e as variáveis: área do curso e ano do curso, sexo, etnia, estado civil, com quem mora, situação laboral, religião, nível socioeconômico, idade e estresse , sendo calculados odds ratios brutos (variável resposta cruzada com uma variável explicativa) e também odds ratios ajustados para todas as variáveis explicativas.

O nível de significância considerado foi de $\mathrm{p}<0,05$, com intervalo de confiança (IC) de 95\%, para todos os testes estatísticos aplicados. As análises estatísticas foram realizadas por programas estatísticos adequados.

Este trabalho orientou-se pela Resolução $n$ 196/96, do Conselho Nacional de Saúde - CNS ${ }^{(21)}$ ligado ao Ministério da Saúde, que define diretrizes e normas que regulamentam as pesquisas que envolvem seres humanos, protegendo os cidadãos participantes da pesquisa em sua integridade física, psíquica e moral. Esta pesquisa foi autorizada pelo Comitê de Ética e Pesquisa da referida universidade com o CAAE: 07535012.5.0000.5495.

\section{RESULTADOS}

A amostra populacional foi caracterizada por 165 alunos participantes da pesquisa. Destes a maioria era do sexo feminino $(64,24 \%)$ e somente $(35,76 \%)$ eram do sexo masculino. A maior parte destes participantes tinha entre 19 e 21 anos. 
Houve a participação de todas as áreas dos diversos cursos existentes na universidade, todavia a maciça participação se deu em torno dos cursos das áreas de biológicas e saúde. A maior parte dos participantes era alunos dos primeiros e segundos anos dos cursos $(67,88 \%)$

A mostra majoritariamente se apresentava na condição de solteiros $(90,30 \%)$ de etnia branca $(86,06 \%)$ e morando com os pais $(61,21 \%)$. Pouco mais da metade exercia uma função laboral $(58,79 \%)$, a maioria se encontrava na faixa de classificação socioeconômica B1 e B2 $(57,58 \%)$ e uma grande parcela se apresentava como tendo alguma crença religiosa $(81,21 \%)$.

A avaliação do uso das substâncias foi feita com o ASSIST, o qual permite, por meio da primeira pergunta, o conhecimento de se o entrevistado já utilizou determinado produto na vida, ainda que em caráter exclusivamente de experimentação; já a segunda e demais questões informam sobre o comportamento em relação à eventual substância nos últimos três meses, ao mesmo tempo que pontuam o padrão de consumo com fins de perscrutar o grau de necessidade de intervenção terapêutica e/ ou a caracterização de abuso ou dependência. Os resultados obtidos para a primeira questão do ASSIST são apresentados na Tabela 1 .

Tabela 1. Distribuição dos universitários em relação ao uso de drogas na vida, segundo os resultados do ASSIST

(Continua)

\begin{tabular}{lcc}
\hline \multirow{2}{*}{ SUBSTÂNCIAS } & \multicolumn{2}{c}{ Amostra $(\mathrm{n}=373)$} \\
& Frequência & Percentual \\
\hline Tabaco & 231 & \\
Não & 142 & 61,82 \\
Sim & & 38,18 \\
Álcool & 2 & \\
Não & 371 & 0,61 \\
Sim & & 99,39 \\
\hline Maconha & 276 & \\
Não & 97 & 73,94 \\
Sim & & 26,06 \\
Cocaína/ crack & 339 & \\
Não & 34 & 90,91 \\
Sim & & 9,09 \\
Anfetaminas/ ecstasy & 353 & 94,55 \\
\hline Não & 20 & 5,45 \\
Sim & &
\end{tabular}

\begin{tabular}{lcc} 
& \multicolumn{2}{c}{ (Conlcusão) } \\
\hline SUBSTÂNCIAS & \multicolumn{2}{c}{ Amostra $(\mathrm{n}=373)$} \\
& Frequência & Percentual \\
\hline Inalantes & 314 & 84,24 \\
$\quad$ Não & 59 & 15,76 \\
$\quad$ Sim & & \\
Hipnóticos/sedativos & 350 & 93,94 \\
$\quad$ Não & 23 & 6,06 \\
$\quad$ Sim & & \\
Alucinógenos & 350 & 93,94 \\
$\quad$ Não & 23 & 6,06 \\
$\quad$ Sim & & \\
Opioides & 369 & 98,79 \\
$\quad$ Não & 04 & 1,21 \\
Sim & & 100 \\
Uso drogas injetáveis & 373 & \\
$\quad$ Não & & \\
\hline
\end{tabular}

Os números apresentados evidenciam grande consumo de álcool, seguido pelo uso do tabaco, com expressão bem menor, mas ainda superior a um terço dos entrevistados, sendo as maiores frequências a seguir ocupadas pela maconha, com pouco mais de um quarto de presença, e os inalantes, com menos de um quinto dos universitários referindo seu consumo. Todas as demais substâncias aparecem com menos de um décimo das ocorrências, sendo o consumo de opioides o menos citado e, por fim, os injetáveis, que não ocorreram na nossa amostra.

As respostas dadas na segunda questão do ASSIST reforçam os problemas do álcool, que apresenta todos os níveis de consumo, desde o uso social, que não necessita intervenção (72,12\%), até a dependência demandando tratamento mais intenso $(3,64 \%)$, passando pelo abuso (24,24\%) que também requer intervenção, embora mais breve. Esses dados evidenciaram também a necessidade de tratamento para usuário de tabaco $(0,61 \%)$, usuário de hipnóticos ou sedativos $(0,61 \%)$ e usuários de maconha $(1,21 \%)$. Chama a atenção que nenhuma das outras substâncias atingiu o patamar de necessidade de tratamento, todavia a necessidade de intervenção breve para os usuários de inalantes $(4,85 \%)$, cocaína $(3,64 \%)$, maconha (10,3\%) e anfetaminas (1,21\%).

Os dados referentes à percepção do estresse, medidos pela EPS-10, estão condensados na Tabela 2 , com a distribuição das frequências em três faixas de pontuação. 
Tabela 2. Distribuição dos universitários por faixa de pontuação conforme a EPS-10

\begin{tabular}{|c|c|c|}
\hline \multirow{2}{*}{ EPS-10 } & \multicolumn{2}{|c|}{ Amostra $(n=373)$} \\
\hline & Frequência & Percentual \\
\hline $1^{a}$ faixa: até 16 pontos & 86 & 23,03 \\
\hline $2^{\mathrm{a}}$ faixa: de 17 até 21 pontos & 136 & 36,36 \\
\hline 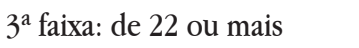 & 151 & 40,60 \\
\hline
\end{tabular}

Consideramos a primeira faixa como de estresse baixo, a segunda como de estresse médio ou moderado e a terceira como de alto nível de estresse.

Como se pode notar, o estresse percebido avaliado pela EPS-10 evidenciou uma ocorrência elevada de alunos na faixa de médio e alto estresse alto.

Para verificar a associação entre as variáveis e consumo de outras drogas, álcool e tabaco foi utilizado o teste estatístico da regressão logística. Nessa análise observou-se uma associação significativa entre o uso de tabaco e não ter religião com associação tanto na regressão logística bruta quanto na ajustada (odds 0,03 e 0,04 , respectivamente); sendo o masculino mais propenso ao fumo em 3,24 vezes na regressão logística ajustada. A regressão ajustada evidenciou uma associação entre morar com a família e o uso de tabaco, condição essa que não era evidente na regressão bruta.

$\mathrm{Na}$ análise entre uso de álcool foi possível perceber uma associação na variável alto estresse, tanto nos dados brutos e também nos ajustados (odds $0,03 \mathrm{e}$ $0,04)$, demonstrando que a pessoa com altos índices de estresse tem 3,75 vezes maiores chances para consumo abusivo do álcool.

Para a verificação da associação entre as variáveis e o consumo de outras drogas, observou-se uma associação significativa, na regressão logística ajustada, entre o consumo de outras drogas e alto estresse os sujeitos com maior pontuação em níveis de estresse, apresentaram 2,35 vezes mais chances de usar drogas que os sujeitos com baixo estresse.

\section{DISCUSSÃO}

A prevalência de 99,39\% de uso de álcool na vida em nossa amostra foi preocupante, seguido em frequência pelo uso de tabaco com $38,18 \%$, depois pelo consumo de maconha com 26,06\% de usuários, inalantes com 15,76\%, cocaína ou crack com $9,09 \%$, alucinógenos e hipnóticos ou sedativos empatados com 6,06\%, anfetaminas ou ecstasy com 5,45\%, e opioides com apenas 1,21\%.

No primeiro levantamento nacional sobre o uso de álcool, tabaco e outras drogas entre universitários ${ }^{7}$, observamos uma distribuição semelhante de uso de drogas na vida na faixa etária de 18 a 24 anos, de $89,3 \%$ para o álcool, $45,5 \%$ para o tabaco e $26,9 \%$ a maconha, ressalvando-se que esses índices de consumo mantiveramse semelhantes nos últimos 12 meses e nos últimos 30 dias. Nesse levantamento ${ }^{7}$, o consumo de inalantes, anfetaminas ou ecstasy, alucinógenos, hipnóticos ou sedativos e opioides foi superior à frequência observada na amostra atual, todavia o consumo de cocaína e derivados foi menor com $6 \%$. A frequência de consumo dos inalantes pode ser pela facilidade de aquisição, quer pelo baixo custo quer pela disponibilidade de grande gama de produtos que podem se prestar a essa utilização, ou também porque a universidade onde foi realizada a pesquisa está próxima de indústria calçadista, onde se usam inalantes e solventes para a confecção dos calçados, ou ainda, por associados também a menor estigmatização dos usuários e menor repressão.

Esta pesquisa mostrou que o consumo de substâncias, exceto álcool e tabaco não exibiu um padrão de uso intenso, não sendo indicada a necessidade de tratamento, segundo os resultados do ASSIST, porém por se tratar de drogas ilícitas já representa um fator de risco que nos leva a pensar, como já indicamos, na importância de prover alguma intervenção que pudesse apresentar impacto na saúde desses indivíduos.

Nesta pesquisa foi evidenciada uma associação entre uso de álcool e o alto estresse percebido. Os principais motivos para que estes índices de estresse se evidenciem, como nos mostra a literatura, está associado às questões acadêmicas e as transformações causadas pelo ingresso na universidade ${ }^{2,14,22}$.

A respeito do tabaco, observou-se na amostra estudada a prevalência de fumantes menor que as encontradas em alguns levantamentos ${ }^{1,7}$, porém com necessidades de tratamento, estimadas pelo ASSIST, em quase $17 \%$ dos usuários. Esse fator é relevante, uma vez que tabagismo é um dos principais responsáveis 
pelas doenças evitáveis e mortes prematuras no mundo, lembrando que cerca de metade dos fumantes morre em consequência de alguma condição associada a esse hábito.

(7) Nesse estudo, verificou-se maior frequência de uso de tabaco entre homens, indivíduos que moravam com os pais e que não possuíam religião. Esses dados também foram encontrados em pesquisa semelhante ${ }^{2}$.

Identificamos, em nosso estudo que cerca de 76\% da amostra apresentou autopercepção de estresse moderado e alto. Em trabalhos recentes realizado com esta mesma população pesquisada, usando a EPS-10, também foi encontrada alta incidência de estresse, principalmente, na faixa mais jovem e no sexo feminino, com diferenças estatisticamente significativas ${ }^{22,23}$.

Ainda a respeito do estresse, agora relacionando-o ao uso de drogas, foram encontradas associações significativas na regressão ajustada. $\mathrm{Na}$ literatura encontramos dados relacionados com população semelhante apontando que o uso de determinadas drogas (como a maconha e cocaína) produzem sensação de relaxamento, o que para muitos seria uma forma de aliviar o estresse. ${ }^{(24)}$ Como alguns autores apontam similaridades entre o estresse e a ansiedade ${ }^{25,26}$, chegando a considerar as condições como sinônimas, talvez fosse interessante avaliar esse aspecto futuramente, posto que uma das justificativas mais comuns para o uso de qualquer das substâncias estudadas é "para aliviar o estresse"

Devemos ressaltar que como estamos trabalhando com uma amostra bastante jovem, não é de estranhar que tenhamos índices elevados de estresse percebido sem ter reflexos sobre a qualidade de vida, por exemplo, pois um dos fatores que modula as consequências do estresse é o tempo de exposição, além do aspecto de que nosso instrumento de avaliação, a EPS-10, parece avaliar o estresse sob a ótica clássica, menos que sob o ponto de vista interativo5.

Resta colocar que a presença dos níveis de estresse que encontramos aponta para a necessidade de disponibilização de mecanismos ou projetos de intervenção que melhor atendam essa população, como já apontado na literatura, para promover saúde mental e mesmo para o diagnóstico e tratamento precoces ${ }^{26,28}$.

\section{CONCLUSÃO}

O consumo de álcool, tabaco e outras drogas desta pesquisa se aproximou do I Levantamento Nacional sobre o Uso de Álcool, Tabaco e Outras Drogas entre universitários das 27 capitais brasileiras; realidade essa bastante constante em todo país.

Dentre os vários fatores de risco para o consumo de álcool, tabaco e outras drogas, o estresse foi percebido como um fator significativo nesta pesquisa, e também em outras como nos mostra a literatura.

Diante destas constataçoes, mais do que nunca se faz necessário pensar em programas de promoção de saúde entre universitários para que estes índices sejam revertidos.

\section{AGRADECIMENTOS}

O presente trabalho foi realizado com apoio da Coordenação de Aperfeiçoamento de Pessoal de Nível Superior - Brasil (Capes) - Código de Financiamento 001

\section{REFERÊNCIAS}

1. Carlini E. supervisão. II Levantamento Domiciliar sobre Uso de Drogas Psicotrópicas no Brasil: estudo envolvendo as 108 maiores cidades do país, 2005. Cebrid/Unifesp/Senad. 2006. Brasília

2. Ferro LRM, Meneses-Gaya C. Resiliência como fator protetor no consumo de drogas entre universitários. Saúde e Pesquisa. 2015;8.

3. Silva ÉC, Tucci AM. Padrão de consumo de álcool em estudantes universitários (calouros) e diferença entre os gêneros. Temas em Psicologia. 2016;24(1):313-23.

4. Jalilian F, Karami Matin B, Ahmadpanah M, Ataee M, Ahmadi Jouybari T, Eslami AA, et al. Sociodemographic characteristics associated with cigarettes smoking, drug abuse and alcohol drinking among male medical university students in Iran. Journal of research in health sciences. 2015;15(1):42-6.

5. Preto VA. O estresse em universitários de enfermagem e sua relação com fatores pessoais e ambientais[tese]. Ribeirão Preto: Universidade de 
São Paulo, Escola de Enfermagem; 2018. 175p.

6. Borba CdS. Investigação dos sintomas de Ansiedade Social nos universitários[dissertação].Manaus: Universidade Federal do Amazonas, Faculdade de Psicologia; 2018. 165p.

7. Brasil. Secretaria Nacional de Políticas sobre Drogas. Andrade AGd, Duarte PdCAV, Oliveira LGd(organizadores). I Levantamento Nacional Sobre o Uso de Álcool, Tabaco e Outras Drogas entre Universitários das 27 Capitais Brasileiras. Grea/IpqHCFMUSP. Brasília: SENAD; 2010

8. Laranjeira R, Madruga C, Pinsky I, Caetano R, Mitsuhiro S, Castello G. II Levantamento Nacional de Álcool e Drogas (LENAD)-2012. São Paulo: Instituto Nacional de Ciência e Tecnologia para Políticas Públicas de Álcool e Outras Drogas (INPAD), UNIFESP. 2014.

9. ONU Organização das Naçoes Unidas. Relatório Mundial sobre Drogas. Escritório das Naçoes Unidas sobre Drogas e Crimes (Unodc). 2014

10. Selye H. The stress of life1956.

11. Lipp MEN. Mecanismos neuropsicofisiológicos do stress: teoria a aplicações clínicas. In.: Mecanismos neuropsicofisiológicos do stress: teoria a aplicações clínicas. 2003.

12. Luft CDB, Sanches SdO, Mazo GZ, Andrade A. Versão brasileira da Escala de Estresse Percebido: tradução e validação para idosos. Revista de Saúde Pública. 2007;41:606-15.

13. Carlotto MS, Câmara SG, Diehl L, Ely K, de Freitas IM, de Azeredo Schneider G. Estressores Ocupacionais e Estratégias de Enfrentamento. Revista Subjetividades. 2018;18(1):92-105.

14. Torquato JA, Goulart AG, Vicentin P, Correa U. Avaliação do estresse em estudantes universitários. InterSciencePlace. 2015;1(14).

15. Yosetake AL, Camargo IMdL, Luchesi LB, GherardiDonato ECS, Teixeira CAB. Estresse percebido em graduandos de enfermagem. SMAD Revista eletrônica saúde mental álcool e drogas. 2018;14(2):117-24.

16. Henrique IFS, De Micheli D, Lacerda RBd, Lacerda LAd, Formigoni MLOdS. Validation of the Brazilian version of alcohol, smoking and substance involvement screening test (ASSIST). Revista da Associacao Medica Brasileira. 2004;50(2):199-206.

17. Siqueira Reis R, Ferreira Hino AA, Romélio R A C. Perceived stress scale: reliability and validity study in Brazil. Journal of health psychology. 2010;15(1):107-14.

18. Trigo M, Canudo N, Branco F, Silva D. Estudo das propriedades psicométricas da Perceived Stress Scale (PSS) na população portuguesa. Psychologica. 2010(53):353-78.

19. André MV. A relação entre qualidade de vida no trabalho e saúde mental do trabalhador para professores de educação infantil em uma escola de Tubarão/SC. Psicologia-Tubarão. 2018.

20. Neves AdSBM. Evidências de Validade da PSS10 e PSS-14: Estudo com Análise Fatorial e de Rede[Dissertação]. Campinas: PUC-Campinas, Programa de Pós Graduação em Psicologia; 2018. $103 p$.

21. Brasil, Saúde MdSCNd. Resolução nº 196/96 sobre pesquisa envolvendo seres humanos. Inf Epidemiol SUS. 1996;5(2).

22. Silva RF, Silva SF, Barbosa TC, Silva M, Quaresma FRP. Nível de Percepção de Estresse e Qualidade de Vida Entre os Técnicos de Enfermagem das Unidades de Pronto Atendimento de PalmasTO. Revista Brasileira de Ciências da Saúde. 2018;22(3):261-6.

23. Führer FM-EC, Lopes DCP, Aguiar PM. Cefaleia e qualidade de vida na graduação de medicina. Revista Brasileira de Neurologia e Psiquiatria. 2015;19(2).

24. Disconzi CMDG, Rodrigues CMC, Corso KB. Avaliação da propensão à síndrome de burnout em estudantes universitários e o uso das estratégias de enfrentamento. Revista Eletronica Gestão \& Saúde. 2018;9(3):419-29.

25. Bonafé FSS, Carvalho JdS, Campos JADB. Depressão, ansiedade e estresse e a relação com o consumo de medicamentos. Psicologia, Saúde \& Doenças. 2016;17(2):105-19.

26. Patias ND, Heine JA, Dell'Aglio DD. Bem-estar subjetivo, violência e sintomas de depressão, ansiedade e estresse em adolescentes. Avaliação 
Psicológica. 2017;16(4):468-77.

27. Gomes IP, Pereira RAdC, Santos BFd, Pinheiro MdA, Alencar CH, Cavalcanti LPdG. Factors Associated with Continued Smoking Addiction and Alcohol Consumption among Medical Students in Capital City in the Northeast of Brazil. Revista Brasileira de Educação Médica. 2019;43(1):55-64.

28. Silva LC, de Afonseca Salles TL. O estresse ocupacional e as formas alternativas de tratamento. Revista de Carreiras e Pessoas (ReCaPe). 2016;6(2).

Recebido em: 19/03/2019

Aceito em: 20/08/2019 